\title{
MARKETING
}




\section{PROPENSÃO DOS CONSUMIDORES AO USO DE SERVIÇOS DE PAGAMENTO EM DISPOSITIVOS MÓVEIS}

CONSUMER'S PROPENSITY TO USE PAYMENT SERVICES ON MOBILE DEVICES

Luis Hernan Contreras Pinochet

Universidade Federal de São Paulo

Priscila Melo de Albuquerque Mota

Universidade Federal de São Paulo

Evandro Luiz Lopes

Universidade Federal de São Paulo

\author{
Data de submissão: 07 ago. 2016. Data de aprovação: \\ 08 mar. 2017 . Sistema de avaliação: Double blind review. \\ Universidade FUMEC / FACE. Prof. Dr. Henrique Cordeiro \\ Martins. Prof. Dr. Cid Gonçalves Filho.
}

\section{RESUMO}

Objetivo deste artigo é analisar a propensão do consumidor ao uso de serviços de pagamentos em dispositivos móveis a partir dos construtos: confiança, facilidade de uso percebida, utilidade percebida, inovação pessoal e autoeficácia. O modelo adaptado a este estudo foi proposto por Duane, $O$ ' Reilly, e Andreev (20|4). A pesquisa, que utilizou abordagem quantitativa com o apoio da técnica de coleta de dados do tipo survey em corte transversal único, obteve os dados oriundos de 365 respondentes que foram analisados por meio da técnica de Modelagem de Equações Estruturais - MEE. Os resultados obtidos demonstraram que todas as hipóteses foram validadas no modelo. $O$ construto autoeficácia, que apesar de influenciar a propensão ao uso de pagamento em dispositivos móveis, apresentou menor carga, dado que a maior parte dos participantes, por pertencerem a grupos de jovens usuários, caracterizam-se por viverem numa "nowconomy", isto é, a busca pelo imediatismo, reduzindo assim, o tempo de utilização das novas tecnologias.

\section{PALAVRAS-CHAVE}

Serviço de Pagamento. Dispositivos Móveis. Disrupção.Tecnologia. Consumidor. 


\section{ABSTRACT}

The aim of this paper is to analyze the consumer's propensity to use payment services on mobile devices based on the constructs: trust, perceived ease of use, perceived usefulness, innovation and personal self-efficacy. The model adapted to this study was proposed by Duane, O'Reilly, and Andreev (2014). This research used quantitative approach with the support of the survey type data collection technique in single cross-section, we obtained the data from 365 respondents were analyzed using Structural Equation Modeling technique - SEM. The results showed that all hypotheses were validated in the model. The self-efficacy construct that despite influencing the propensity to use payment on mobile devices, showed lower load, since most of the participants, because they belong to groups of young users, are characterized by living in a "nowconomy", this is, the search for immediacy, thus reducing the time on the use of new technologies.

\section{KEYWORDS}

Payment Service. Mobile Devices. Disruption. Technology. Consumer.

\section{INTRODUÇÃO}

Cada vez mais popular, o serviço de pagamentos móveis (mobile payment) vem sendo utilizado por ser um meio seguro, rápido e eficaz para realização de transações financeiras. $O$ serviço de pagamentos móveis fornece, a diversos usuários, serviços em tempo real e aumenta o relacionamento entre consumidores, fornecedores e empresas financeiras por meio da liquidação de pagamentos e concessão de créditos (HU; LEE; KOU, 2005; DEWAN; CHEN, 2005).

Em 20I4, um estudo feito pela Pesquisa Nacional por Amostra de Domicílios (PNAD)', divulgada pelo Instituto Brasileiro de Geografia e Estatística (IBGE) mostrou que o acesso à internet por smart- phones superou o acesso por desktops. $\mathrm{O}$ Brasil possui o sexto maior mercado de smartphones do mundo com 38,8 milhões e deve chegar a 2017 com 70,5 milhões de aparelhos em funcionamento. A projeção é que em 2018 o número de smartphones no mundo chegue a 2,56 bilhões, representando $51,7 \%$ do mercado de dispositivos móveis. $O$ resultado é explicado na pesquisa conduzida pelo IBGE por dois fatores: a cultura e o ambiente regulatório.

Dentre os brasileiros que acessam a internet, $66 \%$ o fazem por meio de smartphones (Pesquisa Brasileira de Mídia², 2015).A pesquisa revelou ainda que os usuários de aplicativos móveis no país passam 17 milhões de horas diariamente em aplicativos, sendo a maior parte desses (44\%), aplica-

\footnotetext{
Pesquisa Nacional por Amostra de Domicílios Contínua (PNAD, População) Disponível em: http://www.ibge.gov.br/home/estatistica/pesquisas/pesquisa_resultados. php?id_pesquisa=|49:Acesso em 15/07/2016.

2 Pesquisa Brasileira de Mídias (2015). Yahoo mapeia uso de dispositivos móveis pelos brasileiros, Revista Pronews, 20I6. Disponível em: https://revistapronews.wordpress.com/2016/04/07/yahoo-mapeia-uso-de-dispositivos-moveis-pelos-brasileiros/.Acesso em: 02/05/2016.
} 
tivos de produtividade. Entre 2014 e 2015 os aplicativos móveis de estilo de vida e compras cresceram $72 \%$.

Dispositivos móveis podem ser entendidos como tecnologias que não necessitam de um determinado espaço para seu funcionamento, como aparelhos celulares, notebooks, tablets, entre outros. O smartphone se diferencia dos aparelhos celulares comuns por possuir um sistema operacional que funciona com aplicações terceiras que podem ser obtidas por meio de uma loja de aplicativos (BROWN;VENKATESH, 2005; THEOHARIDOU; MYLONAS; GRITZALDIS, 20I2).

Ao observarmos a literatura científica nos últimos cinco anos, identificou-se poucos estudos que buscaram analisar a propensão do consumidor ao uso de serviços de pagamentos em dispositivos móveis, sendo os trabalhos "Mobile Payment Market and Research - Past, Present and Future" (DALHBERG et al., 2006), "Extending UTAUT2 To Explore Consumer Adoption Of Mobile Payments" (SLADE; WILLIAMS; DWIVDEI, 20I3) e "Trends in the Use of Mobile Payment in Brazil:An Analysis Using the Delphi Method" (PEREZ et al., 2013) os mais relevantes.

Dentro desta perspectiva, o modelo de Duane, O' Reilly e Andreev (20I4) publicado no Behaviour \& Information Technology (BIT) tem se destacado na literatura medindo a percepção dos consumidores Irlandeses no uso de smartphones para serviços de pagamentos móveis. Porém, quando se verifica as pesquisas realizadas no Brasil, apesar de alguns esforços, notamos uma lacuna em relação a profundidade e amplitude dos estudos desta temática (PEREZ et al., 20I3).

Esse trabalho busca contribuir com o entendimento dessa recente temática com a replicação de uma pesquisa que se propôs a analisar o uso de aplicativos de dispositivos móveis (sendo considerados smartphones e tablets). Portanto, o objetivo da pesquisa é analisar a percepção do consumidor em relação a propensão ao uso de serviços de pagamento em dispositivos móveis.

$O$ artigo possui a seguinte estrutura: introdução, referencial teórico o qual fornece base conceitual do tema, na sequência são apresentados: a construção do modelo teórico no método, a análise de dados e, ao final, as conclusões.

\section{REFERENCIALTEÓRICO E HIPÓTESES}

Neste tópico serão apresentados os conceitos teóricos e os estudos que fundamentaram esta pesquisa. Com o intuito de apresentar uma estrutura que facilite $o$ entendimento abordado, o referencial teórico foi subdividido em: a percepção do consumidor, avanço da tecnologia e os tipos de pagamentos, os construtos que levam a propensão ao uso de pagamento em dispositivos móveis.

\section{A percepção do consumidor}

Os meios de pagamento acompanharam a evolução da humanidade. $O$ papel moeda antes amplamente utilizado foi substituído por cheques, depois cartões magnéticos, telefone e por dispositivos com internet, com os pagamentos móveis utilizando celulares, tablets, entre outros (GUPTA; XU; ZHANG, 20I I).A rotina cada vez mais acelerada trouxe a necessidade do consumidor obter acesso em tempo real a serviços que antes demandavam um deslocamento e uso maior do tempo, possibilitando maior mobilidade nas atividades de negócios.

As razões que podem motivar ou desmotivar o usuário a aceitação dos serviços 
de pagamentos móveis podem ser intrínsecas, relacionadas a conveniência que fornecem ao cliente, ao tempo, se realmente a transação reduz o tempo que levaria sem o uso de dessa tecnologia e a facilidade de uso. Portanto, o nível de desconforto do usuário ou da falta de controle pode influenciar negativamente, levando-o a se sentir subjugado a tecnologia. A insegurança em relação aos dados bancários e pessoais do cliente também podem se tornar uma barreira ao uso dessa tecnologia.

\section{Avanço tecnológico e tipos de pagamentos}

A tecnologia trouxe para a vida das pessoas possibilidades de comprar on-line sem precisar se locomover até uma loja. Grandes redes varejistas possuem a opção de compra pelo canal on-line ou em loja física, ou ainda de comprar pelo website e posteriormente, apenas realizar a retirada na loja. É o futuro chamado omnichannel no qual as distinções entre loja física e on-line não vão mais existir (BRYNJOLFSSON; HU; RAHMAN, 20I3).

Para efetuar essas transações e ter o poder de comprar aonde quer que esteja, o consumidor conta com o auxílio da tecnologia móvel. Os meios de pagamentos móveis tornam-se importantes, facilitando os pagamentos do comércio eletrônico e de negócios móveis, ao invés do uso de dinheiro físico (SACCOL; REINHARD, 2007).

As principais tecnologias utilizadas remotamente ou por aproximação são: o Token ID, o NFC (Near Fiel Communication), - SMS, o QR-Code e o celular como ponto de venda. O Token ID é um dispositivo de segurança que gera uma senha pessoal temporária para uma operação. $O$ usuário cria uma conta e vincula seu cartão de crédito ao número de celular, quando fizer uma compra insere o número do telefone móvel e em seguida recebe uma mensagem com um token que deve ser completado com o código de segurança do cartão para finalizar a transação. Para cada compra é enviado um token diferente (AKYILDIZ; KASIMOGLU, 2004; UZUREAU; NEWTON, 20II). O QR-Code também surge como um sistema que codifica informação de um pequeno espaço gerando uma imagem que pode ser lida. $O$ usuário digita seus dados em um programa de autenticação e é gerada uma imagem que é capturada pelo celular. No celular como ponto de venda um dispositivo leitor de cartões é colocado no celular e permite a realização de transações débito e crédito.

\section{Construtos associados com pagamentos em serviços móveis}

Para que os pagamentos móveis tenham sucesso, o consumidor deve entende-los como sendo úteis, seguros e fáceis de utilizar (VIEHLAND;YOONG LEONG, 20I0). Neste contexto são apresentados os construtos associados com pagamentos em serviços móveis que nortearam esta pesquisa.

\section{Confiança}

O sentimento de confiança dos usuários para um serviço on-line é um determinante importante ao considerar a sua propensão ao uso (CHAU et al., 2007; WU; TSANG, 2008; ROCA; GARCIA; VEGA, 2008). A confiança é o mais importante antecedente (SANCHEZ- FRANCO; RONDAN - CATALUÑA, 20II).

A confiança é crucial no comércio eletrônico, dado o anonimato nas interações entre comprador-vendedor e a falta de contratos e acordos formais.A falta de con- 
fiança é um grande obstáculo na adoção de serviços móveis (DAHLBERG et al., 2008; VARNALI;TOKER, 2009). A confiança possui uma reflexão em diferentes dimensões para a compreensão do seu alcance (HSU; KULVIWAT, 2006; HSU; JU; CHANG, 2007; ROCA; GARCIA;VEGA, 2008; CHIU et al., 2009; CHEN; CHEN; YEN; 20I I). Níveis mais elevados de compromisso ético percebido também aumentam a confiança e influenciam fortemente as decisões de compra on-line (YANG, CHANDLREES; CHAO, 2009; CLEFF, 2007; SANCHEZ-FRANCO; RONDAN-CATALUÑA, 20II).

Portanto, é fundamental que um regulador objetivo independente e que $\circ$ governo desempenhe um papel central no estabelecimento de legislação e padrões de serviço em aplicativos móveis. Os "vendedores on-line" podem minimizar a incerteza mediante uma exibição clara de suas regras e todos os aspectos e vedações legais necessárias de aprovação. Nesse sentido, verifica-se a seguinte hipótese proposta:

HI: A confiança do consumidor tem impacto positivo sobre a propensão de fazer pagamentos móveis utilizando dispositivos móveis.

\section{Inovação Pessoal}

A inovação pessoal no domínio das TICs (Tecnologias da Informação e Comunicação) caracteriza a adoção da tecnologia, que é definida como a disposição de um indivíduo para experimentar qualquer nova tecnologia (AGARWAL; PRASAD, 1998).

Inovação pessoal é específica para um indivíduo, que é o mesmo da inovação inata, que é parte da personalidade de um indivíduo (IM; KIM; HAN, 2008). Inovação inata teve um impacto positivo na condução do consumidor em aceitar a mobilidade como ferramental de marketing (BAUER et al., 2005; GUPTA; XU; ZHANG, 20I I).

A inovação pessoal no domínio das TICs é um importante estímulo influenciando percepções de serviços da internet por meio da tecnologia móvel, e que a inovação pessoal no domínio das TICs poderá influenciar tanto utilidade percebida quanto na facilidade de uso percebida, esse último sendo particularmente afetado. Portanto, as seguintes hipóteses foram definidas:

H2a: A inovação pessoal tem impacto positivo sobre a percepção da facilidade dos consumidores de fazer pagamentos móveis utilizando dispositivos móveis.

H2b: A inovação pessoal tem impacto positivo sobre a propensão dos consumidores de fazer pagamentos móveis utilizando dispositivos móveis.

H2c: A inovação pessoal tem impacto positivo sobre a percepção da utilidade dos consumidores de fazer pagamentos móveis utilizando dispositivos móveis.

\section{Facilidade de uso percebida}

Facilidade de uso tem sido documentada como sendo um condutor iminente da aceitação de aplicativos móveis (DAHLBERG et al., 2008; VIEHLAND; YOONG LEONG, 20I0).

A facilidade de uso se torna ainda mais importante para pagamentos de serviços móveis, que competem com soluções de pagamento estabelecidas, e, portanto, precisam oferecer benefícios quando se trata de facilidade de uso. Por conseguinte, uma das principais razões para a lenta difusão de pagamentos móveis em particular pode ser uma falha na compreensão da percepção da facilidade de uso dos consumidores 
fazerem os pagamentos móveis utilizando celulares (SCHIERZ; SCHILKE; WIRTZ, 2010). Assim, verifica-se a seguinte hipótese proposta:

H3a: Facilidade de uso percebida terá um efeito positivo sobre a propensão dos consumidores para usar dispositivos móveis para fazer pagamentos móveis.

\section{Utilidade percebida}

A utilidade percebida tem sido consistentemente apresentada na literatura como elemento preditor relacionado com a intenção comportamental, mas para o contexto de consumo, como busca dos benefícios e necessidades. Normalmente são explicados pelas teorias da motivação para entender o que levava o consumidor a utilizar ou realizar algo (BROWN;VENKATESH, 2005).

Pesquisas afirmam que a utilidade percebida impacta positivamente 0 consumidor em relação a propensão ao uso de pagamentos móveis (DAHLBERG et al., 2008). Nesse sentido, verifica-se a seguinte hipótese proposta:

H3b: Utilidade percebida terá um efeito positivo sobre a propensão dos consumidores para usar dispositivos móveis para fazer pagamentos móveis.

\section{Autoeficácia}

A autoeficácia funciona como um regulador cognitivo de ação no qual as pessoas que se sentem mais seguras em executar uma atividade possuem conhecimentos ou habilidades necessárias para realizá-la. Assim, pessoas ou consumidores com maior autoeficácia se sentiriam mais confortáveis e seguras com seus conhecimentos para realizar compras on-line (YOUNG HOO; KIM; YUJONG, 2009; HSU; CHANG; YEN, 20I I; LEE; HSIEH; HUANG, 20I I).
No entanto, é preciso reconhecer que - mercado de pagamentos móveis ainda está em sua infância apesar de sua potencialidade, e nesse sentido, os modelos de pagamentos móveis trazem oportunidades para um mercado lucrativo, em que existem serviços rivais que continuarão a competir uns com os outros e investir quantias significativas de recursos financeiros em aquisições e pesquisas (ONDRUS; LYYTINEN, 20I I).

Assim, diferentes vendedores on-line adotam diferentes modelos de pagamentos móveis e, consequentemente, os consumidores atualmente interagem com vários modelos de pagamentos móveis e continuarão a fazê-lo no futuro previsível (ONDRUS; LYYTINEN; PIGNEUR, 2009). Baseadas nestas afirmações, as seguintes hipóteses são formuladas:

H4a: Autoeficácia móvel impacta positivamente na facilidade de uso percebida pelos consumidores no uso de dispositivos móveis para fazer pagamentos móveis.

H4b: Autoeficácia móvel impacta positivamente sobre a propensão dos consumidores a usar dispositivos móveis para fazer pagamentos móveis.

\section{MÉTODO}

Este tópico apresenta o design do estudo, contendo a definição do perfil dos participantes da pesquisa, as escalas e medidas, os procedimentos de coleta e de tratamento dos dados.

\section{Participantes, instrumento de coleta, procedimentos e tratamento dos dados}

A população analisada foi constituída de usuários de aplicativos disruptivos em 
dispositivos móveis em maior ou menor grau que possuem acesso a internet. Os dados foram coletados por conveniência, resultando em técnica de amostragem não probabilística, limitando, portanto, a generalização dos resultados desta pesquisa (MALHOTRA, 20I4).

O presente estudo foi desenvolvido tendo como base dados coletados por meio de uma survey, replicando e adaptando o estudo do modelo de Duane, O' Reilly e Andreev (2014) para a realidade brasileira. Esse instrumento de coleta de dados foi composto por 28 (vinte e oito) assertivas relacionadas em 06 (seis) variáveis latentes de categoriais distintas, que foram respondidas por meio de uma escala Likert, com end points ancorados em I=discordo totalmente e 5=concordo totalmente (vide Quadro I). Há ainda, questões demográficas, e questões referentes ao uso de dispositivos para pagamentos móveis que foram analisadas pelo software IBM SPSS Statistics Version 20.

$O$ instrumento de coleta de dados compreendeu um questionário estruturado baseado em aspectos teóricos, no qual foi dividido e agrupado em variáveis latentes, ou seja, questões que tratam de um mesmo aspecto conforme Parasuraman, Zeithaml e Berry (1985).

A coleta de dados foi composta por meio da técnica de questionário eletrônico, que foi auto-aplicado via internet (site de pesquisa Question Pro) no qual o link de acesso foi disponibilizado pelo Facebook e e-mail, enquanto que a pesquisa com questionário impresso foi conduzida em faculdades, locais públicos ou em ambiente de fácil acesso ao sujeito de pesquisa. Os dados foram coletados entre os meses de abril e maio de 2016.

Ao todo foram obtidos 405 questioná- rios, com a exclusão de 40 questionários inválidos por preenchimento incorreto ou variáveis que apresentassem mais que $20 \%$ dos campos em branco apresentando missings, assim, foi obtido uma amostra final de 365 respondentes. $O$ software G*Power 3.I foi utilizado a priori, para estimar o tamanho da amostra.

De acordo com as orientações de Faul (2009), foi identificada a variável latente que recebe maior número de setas, no qual se busca entender o objetivo da pesquisa, no caso deste estudo, a variável latente "Propensão ao pagamento móvel" com cinco variáveis independentes ligadas a ele. Utilizando os parâmetros de tamanho do efeito $\left(\mathrm{f}^{2}\right)$ igual a 0,15 e poder de teste igual a 0,80, indicados por Hair, Black, Babin \& Anderson (2010), além de significância ao nível de $10 \%(p<0,10)$, o resultado é um $F$ crítico de 2,32, com amostra mínima de 92 indivíduos. Nesta pesquisa, com uma amostra de 365 indivíduos, tamanho do efeito $f^{2}$ igual a 0,15 e 5 preditores, F crítico 2,23, o poder de teste ( 1 - $\beta$ err prob) é igual a 0,99 .

O estudo teve abordagem quantitativa, de natureza confirmatória. Utilizou-se nesta pesquisa o tipo cross-section (cortetransversal), uma vez que os dados para esta fase foram coletados nos meses de abril e maio de 2016 .

Devido à constatação da presença de distribuição não normal nos conjuntos de dados de diversas variáveis da amostra, optou-se pela utilização do approach Partial Least Squares Structural Equation Modeling (PLS-SEM), levando então ao uso do aplicativo SmartPLS 3 Professional.

\section{ANÁLISE DE DADOS}

Esta seção apresenta a análise descritiva 
QUADRO 1 - Variáveis latentes, indicadores e descrições

\begin{tabular}{|c|c|c|}
\hline Variável latente & Indicador & Descrição \\
\hline \multirow{3}{*}{$\begin{array}{l}\text { Facilidade de uso } \\
\text { percebida }\end{array}$} & FUP1 & No geral, eu acho fácil de usar serviços de pagamentos móveis. \\
\hline & FUP2 & O uso de serviços de pagamentos móveis não requer muito conhecimento. \\
\hline & FUP3 & O uso de serviços de pagamentos móveis não requer muita habilidade técnica. \\
\hline \multirow{4}{*}{ Utilidade percebida } & UP1 & $\begin{array}{l}\text { O uso de serviços de pagamentos móveis pode diminuir o tempo necessário para mi- } \\
\text { nhas atividades. }\end{array}$ \\
\hline & UP2 & $\begin{array}{l}\text { O uso de serviços de pagamentos móveis pode aumentar meu rendimento de acordo } \\
\text { com a quantidade do meu esforço. }\end{array}$ \\
\hline & UP3 & $\begin{array}{l}\text { O uso de serviços de pagamentos móveis melhora a eficácia das minhas atividades de } \\
\text { trabalho. }\end{array}$ \\
\hline & UP4 & $\begin{array}{l}\text { O uso de serviços de pagamentos móveis melhora a eficácia das minhas atividades } \\
\text { pessoais. }\end{array}$ \\
\hline \multirow{3}{*}{ Inovação pessoal } & IP1 & Eu gosto de experimentar os novos serviços de pagamentos móveis. \\
\hline & IP2 & $\begin{array}{l}\text { Entre os meus colegas, eu normalmente sou o primeiro a tentar um novo serviço de } \\
\text { pagamento móvel. }\end{array}$ \\
\hline & IP3 & $\begin{array}{l}\text { Meus colegas levam em consideração minha opinião na hora de usar serviços de pa- } \\
\text { gamentos móveis. }\end{array}$ \\
\hline \multirow{7}{*}{ Autoeficácia } & AE1 & Eu me sinto confiante utilizando serviços de mídia móveis para atividades sociais. \\
\hline & AE2 & $\begin{array}{l}\text { Eu me sinto confiante utilizando serviços de mídia móveis para acessar filmes e músicas } \\
\text { on-line. }\end{array}$ \\
\hline & AE3 & $\begin{array}{l}\text { Eu me sinto confiante utilizando serviços de mídia móveis para acessar mídias de tele- } \\
\text { visão e notícias. }\end{array}$ \\
\hline & AE4 & Eu me sinto confiante utilizando serviços de mídia móveis para acessar mídia impressa. \\
\hline & AE5 & $\begin{array}{l}\text { Eu me sinto confiante utilizando serviços de mídia móveis para assistir programas de } \\
\text { televisão. }\end{array}$ \\
\hline & AE6 & $\begin{array}{l}\text { Eu me sinto confiante utilizando meus serviços de mídia móveis para acessar serviços } \\
\text { de games. }\end{array}$ \\
\hline & AE7 & Eu me sinto confiante utilizando meus serviços de mídia móveis para mídias sociais. \\
\hline \multirow{7}{*}{ Confiança } & $\mathrm{C} 1$ & $\begin{array}{l}\text { O quadro legal para serviços de pagamentos móveis é suficientemente robusto para } \\
\text { proteger os consumidores. }\end{array}$ \\
\hline & $\mathrm{C} 2$ & $\begin{array}{l}\text { Eu acredito que os provedores de serviços de pagamentos móveis têm experiência e } \\
\text { recursos suficientes para fornecer esses serviços. }\end{array}$ \\
\hline & $\mathrm{C} 3$ & $\begin{array}{l}\text { Eu acredito que os provedores de serviços de pagamentos móveis agirão eticamente } \\
\text { quando a captura, retenção, processamento e gerenciamento dos meus dados pes- } \\
\text { soais. }\end{array}$ \\
\hline & $\mathrm{C} 4$ & $\begin{array}{l}\text { Eu acredito que os provedores de serviços de pagamentos móveis agem honestamente } \\
\text { ao lidar com os consumidores. }\end{array}$ \\
\hline & $\mathrm{C} 5$ & $\begin{array}{l}\text { Estou confiante com os controles de privacidade dos provedores de serviços de paga- } \\
\text { mentos móveis. }\end{array}$ \\
\hline & C6 & $\begin{array}{l}\text { Eu acredito que todos os prestadores de serviços de pagamentos móveis implementam } \\
\text { medidas de segurança adequadas para proteger meus dados pessoais. }\end{array}$ \\
\hline & $\mathrm{C} 7$ & $\begin{array}{l}\text { Órgãos reguladores para prestadores de serviços de pagamentos móveis tem autoridade } \\
\text { suficiente para regular provedores de pagamentos móveis. }\end{array}$ \\
\hline \multirow{4}{*}{$\begin{array}{l}\text { Propensão ao uso de } \\
\text { pagamentos móveis }\end{array}$} & PG1 & $\begin{array}{l}\text { Considero que é seguro fazer um pagamento móvel através da minha operadora de } \\
\text { rede móvel utilizando de pagamentos móveis. }\end{array}$ \\
\hline & PG2 & $\begin{array}{l}\text { Considero que é seguro fazer um pagamento móvel através de uma empresa terceira } \\
\text { utilizando serviços de pagamentos móveis. }\end{array}$ \\
\hline & PG3 & Considero que é seguro fazer um pagamento móvel com meu cartão de crédito. \\
\hline & PG4 & $\begin{array}{l}\text { Considero que é seguro fazer um pagamento com meu cartão de laser utilizando de } \\
\text { pagamentos móveis. }\end{array}$ \\
\hline
\end{tabular}

Fonte: Adaptado de Duane, O’ Reilly e Andreev (2014, p.324). 
das características dos respondentes, bem como mutivariada com o apoio da modelagem de equações estruturais.

\section{Características dos respondentes}

As características dos respondentes desta pesquisa são aqui apresentadas para possibilitar a contextualização da realidade socioeconômica dos participantes deste estudo.Analisando os dados demográficos dos respondentes verifica-se que, grande parte dos respondentes são do gênero feminino e mesmo se tratando de uma amostra não probabilística, suas características estão alinhadas ao público-alvo que utilizam serviços de pagamentos móveis: $29,3 \%$ dos respondentes tem idade entre 22 e 26 anos, $26,3 \%$ possui renda familiar de 4 a 6 salários mínimos e $48,5 \%$ estão cursando graduação.

\section{Propensão ao uso das tecnologias para serviços de pagamentos}

Nesse tópico são apresentados os dados em relação as principais tecnologias utilizadas pelos serviços de pagamento em dispositivos móveis escolhidos pelos respondentes.

O Gráfico I apresenta a propensão ao uso das tecnologias de acordo com o gênero (o usuário poderia escolher mais de uma opção de tecnologia de uso em serviços de pagamento móvel). Em todas as alternativas o gênero feminino se destacou em relação ao masculino, sendo a diferença maior no uso de SMS que foi de $21,07 \%$ para o público feminino e II,7I\% do masculino, e a menor diferença no NFC sendo $20,07 \%$ para as mulheres e $18,73 \%$ para os homens.

A propensão ao uso de tecnologias de acordo com as dez principais ocupações respondidas nos questionários apresentou que a maior parte dos respondentes são usuários do Token ID, no qual os respondentes se caracterizaram de forma geral, por bancários, assim como a tecnologia $N F C$. Já o $S M S$ e o $Q R C o d e$ são mais utilizados por estudantes.

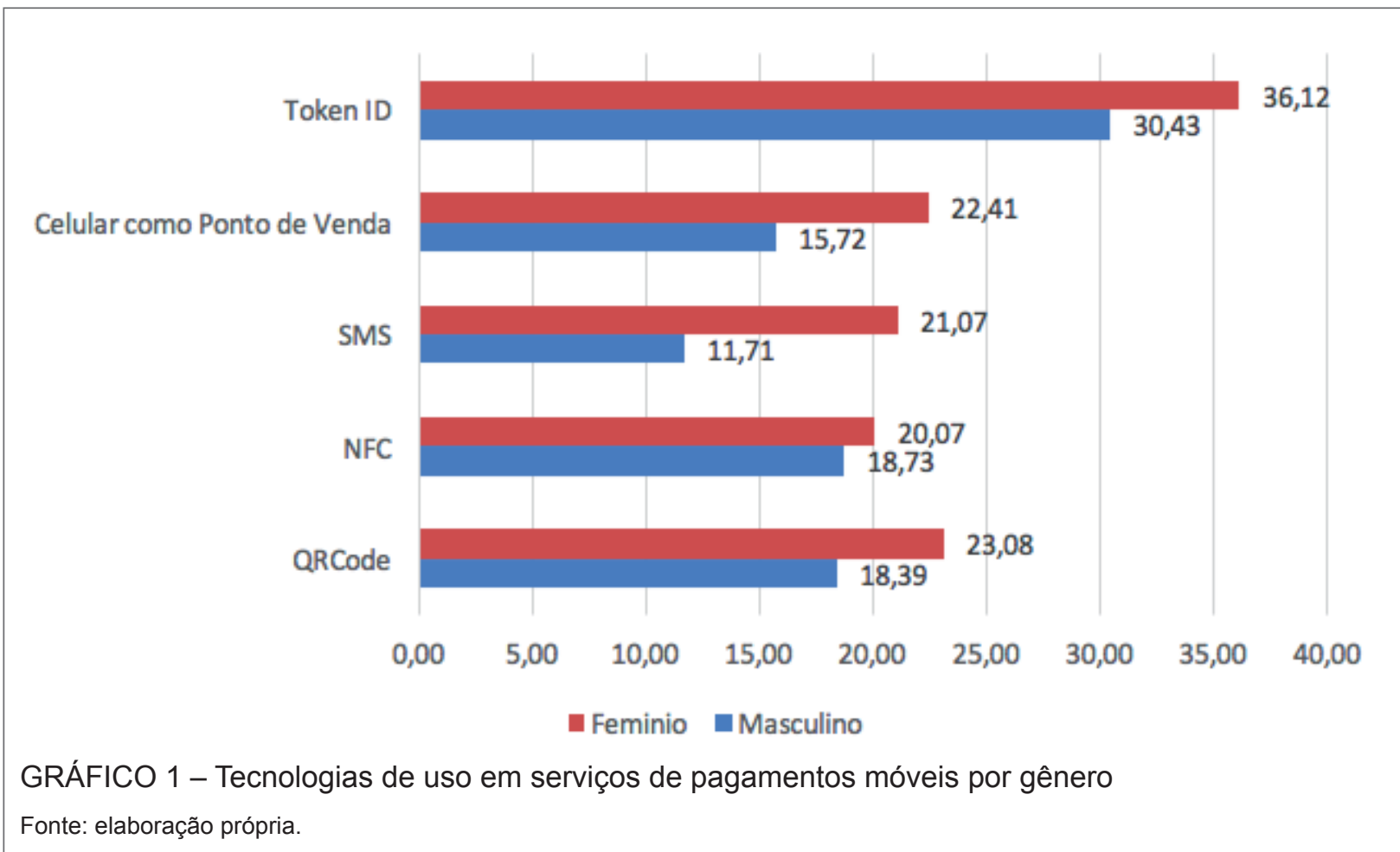




\section{Premissas para o uso da modelagem de equações estruturais}

Nas técnicas de análise multivariada que utilizam variáveis métricas e testes estatísticos, a normalidade multivariada é a condição fundamental de aplicação. A normalidade dos dados foi verificada por meio de observação da curtose e assimetria presente na amostra de dados pelo teste Komolgorov-Smirnov e o respectivo $p$-value de cada variável. Este procedimento foi necessário para limitar a possibilidade da utilização de algumas técnicas de análise estatística que tenham como característica a distribuição normal dos dados.

Portanto, nas variáveis preditoras relacionadas com a "propensão ao pagamento móvel" sobre a propensão ao uso de serviços de pagamento em dispositivos móveis foi possível acomodar a multicolinearidade no modelo porque todos os valores obtidos dos fatores de inflação das variâncias (VIFs) foram menores que 5 , sendo que o indicador que obteve o menor valor foi UPI $(I, 553)$ e maior valor foi C2 $(2,94 I)$ (HAIR et al., 20I0).

Todos os p-value do teste referente aos indicadores resultaram significativos com $p<0,0$ I. Ademais, o resultado do teste de normalidade corroborou pela estimação do modelo estrutural ao analisar a correlação das variáveis em mínimos quadrados parciais.

\section{Análise do modelo estrutural}

Após a primeira iteração, no qual são apresentados os resultados das cargas fatoriais obtidas por cada uma das variáveis latentes, observou-se que todos os valores das cargas fatoriais de cada um dos indicadores, apresentaram valores acima de 0,5. Neste caso, não foi necessário eliminar in- dicadores apresentando como resultado a adequação do modelo.

A validade discriminante avalia se os itens que refletem o fator não estão correlacionados com outros fatores, a validade discriminante fica demonstrada quando, as variâncias extraídas médias forem superiores ou iguais ao quadrado da correlação entre os fatores. $\mathrm{Na}$ Tabela I a seguir observa-se que todas as variâncias extraídas médias são superiores ou igual ao quadrado da correlação entre os fatores, assim, não foi necessária a eliminação de itens do modelo de mensuração.

A análise do modelo de mensuração deve preceder a análise das relações entre as variáveis latentes. $O$ próximo passo foi examinar as variâncias das médias extraídas (AVE) e as correlações quadráticas entre os construtos, a validade convergente, e a confiabilidade composta.

Dado que todas as variáveis de um questionário utilizam a mesma escala de medição, o coeficiente é calculado a partir da variância dos itens individuais (Tabela 2). Os alphas de cronbach variaram entre 0,692 e 0,901 . Sendo que valores acima de 0,60 a 0,75 são considerados moderados, e de 0,75 a 0,90 altos (STRIENER, 2003; MALHOTRA, 20I4).

Segundo Marôco (20I0), a confiabilidade composta consiste na avaliação realizada a partir dos resultados obtidos do modelo de análise fatorial confirmatória para os coeficientes de mensuração e dos erros de medida. Assim, as confiabilidades compostas variaram entre 0,824 e 0,922 , o que é considerado adequado.

Para este modelo as AVEs variaram entre 0,520 e 0,726. Utilizou-se o critério de Fornell e Larcker (198I), isto é, os valores das AVEs devem ser maiores que 0,50. Nes- 
TABELA 1 - Teste de discriminância de Fornell-Larcker

\begin{tabular}{l|c|c|c|c|c|c}
\hline & $\mathbf{( 1 )}$ & $\mathbf{( 2 )}$ & $\mathbf{( 3 )}$ & $\mathbf{( 4 )}$ & $\mathbf{( 5 )}$ & $\mathbf{( 6 )}$ \\
\hline (1) Autoeficácia & $\mathbf{0 , 7 2 1}$ & & & & & \\
\hline (2) Confiança & 0,428 & $\mathbf{0 , 7 9 3}$ & & & & \\
\hline (3) Facilidade de Uso Percebido & 0,338 & 0,423 & $\mathbf{0 , 7 8 1}$ & & & \\
\hline (4) Inovação Pessoal & 0,371 & 0,413 & 0,240 & $\mathbf{0 , 8 5 2}$ & & \\
\hline (5) Propensão PgMóvel & 0,450 & 0,727 & 0,427 & 0,472 & $\mathbf{0 , 8 2 4}$ & \\
\hline (6) Utilidade Percebida & 0,417 & 0,351 & 0,312 & 0,513 & 0,434 & $\mathbf{0 , 7 7 4}$ \\
\hline
\end{tabular}

Nota: a diagonal em destaque apresenta as raízes quadradas da AVE.

Fonte: elaboração própria.

TABELA 2 - AVE, Alpha de Cronbach, Confiabilidade composta, R Square e número de itens

\begin{tabular}{l|c|c|c|c|c|c}
\hline & $\mathbf{( 1 )}$ & $\mathbf{( 2 )}$ & $\mathbf{( 3 )}$ & $\mathbf{( 4 )}$ & $\mathbf{( 5 )}$ & $\mathbf{( 6 )}$ \\
\hline AVE & 0,520 & 0,630 & 0,609 & 0,726 & 0,680 & 0,598 \\
\hline Alpha de Cronbach & 0,845 & 0,901 & 0,692 & 0,813 & 0,843 & 0,772 \\
\hline Confiabilidade Composta & 0,883 & 0,922 & 0,824 & 0,888 & 0,895 & 0,855 \\
\hline R Square & & & 0,130 & & 0,594 & 0,263 \\
\hline$N^{\circ}$ de itens & 7 & 7 & 3 & 3 & 4 & 4 \\
\hline
\end{tabular}

Nota: (1) Autoeficácia; (2) Confiança; (3) Facilidade de Uso Percebido; (4) Inovação Pessoal; (5) Propensão ao Pagamento Móvel; (6) Utilidade Percebida.

Fonte: elaboração própria.

se sentido, todas as variáveis latentes apresentaram variância média extraída maior que $50 \%$, o que atinge os critérios de Chin (1994) e Hair et al. (2010) para a indicação da existência de validade convergente.

Já o $R^{2}$ value mensura a acurácia preditiva do modelo, representando os efeitos combinados das variáveis endógenas sobre as variáveis exógenas. No presente estudo, o $R^{2}$ value demonstrou que o modelo possui acurácia e relevância preditiva em relação ao construto "Facilidade de uso percebida" $(0,130)$, "Propensão ao pagamento móvel" $(0,594)$ e "Utilidade Percebida" $(0,263)$ o que representa, acurácia preditiva substancial analisando as variáveis apresentadas.

Conforme a Figura I, o modelo inicial não necessitou novas iterações em função de todos os indicadores apresentarem valores da AVE superiores a 0,5. Trata-se de um modelo reflexivo, no qual a direção de relação entre variáveis posiciona-se dos construtos (variáveis latentes) - para os indicadores

A construção de diagramas de caminhos (path diagrams) das relações estabelecidas expressão gráfica de causa e efeito estabelecida no modelo teórico, de forma a permitir a visualização dos relacionamentos derivados dos construtos. Este diagrama permite descrever as relações de causa e efeito identificadas nas relações entre variáveis dependentes - também chamadas de endógenas e as variáveis independentes - identificadas como exógenas, além de verificar os relacionamentos entre os construtos.

$\mathrm{Na}$ aplicação prática da modelagem de equações estruturais para o modelo proposto, a abordagem sugere a avaliação dos modelos de mensuração individualmente, buscando verificar sua validade e consistência e, em seguida, realizar a abordagem do modelo estrutural. A partir de uma amostra, desenvolvem-se 


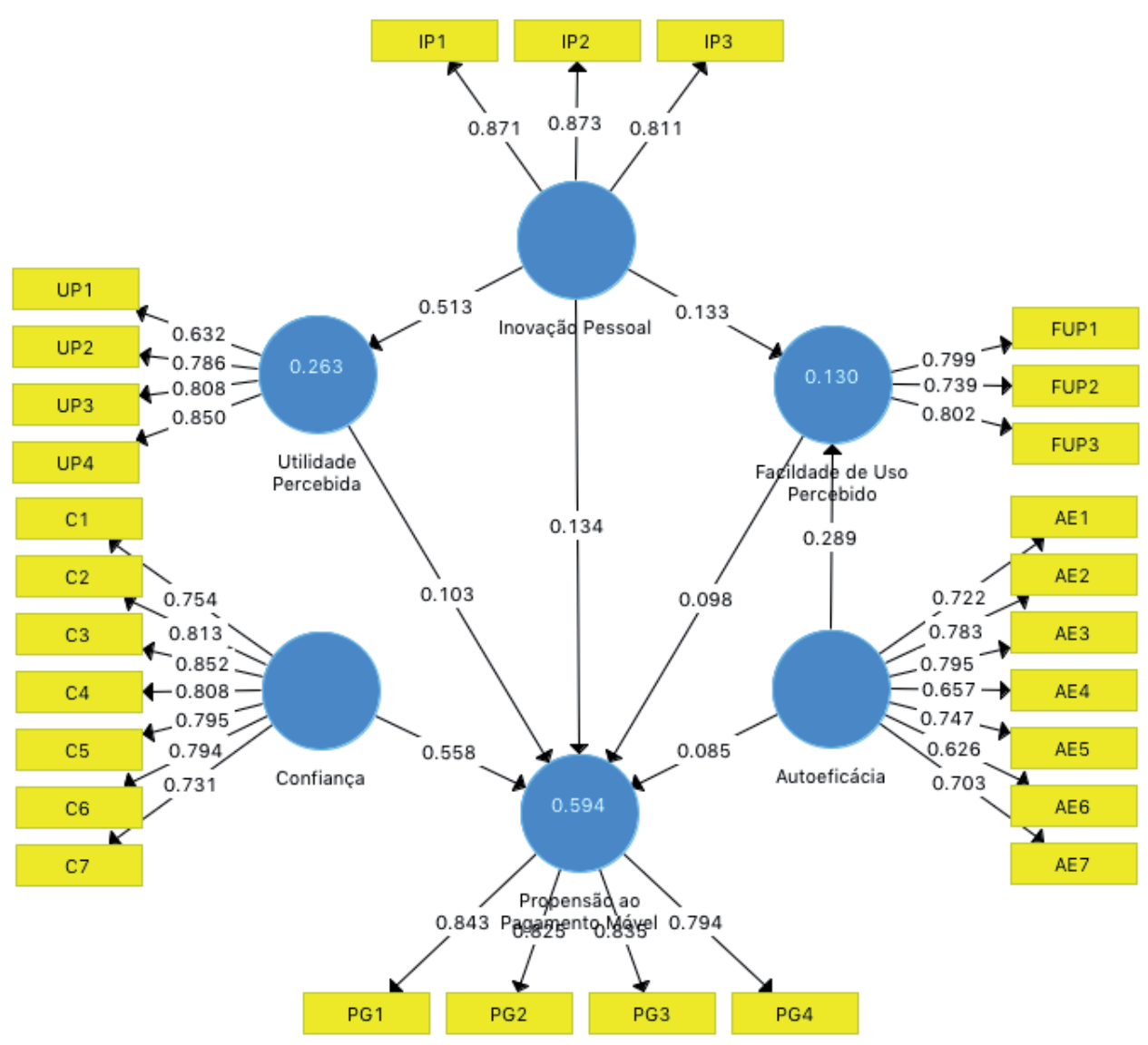

FIGURA 1 - Modelo da validade convergente em relação a propensão o uso dos serviços de pagamento em dispositivos móveis

Fonte: Elaboração própria.

outras amostras compostas de elementos da amostra original e estimam-se os parâmetros do modelo nestas amostras (BROWNE; CUDECK, 1993).

O modelo proposto nesta pesquisa foi estimado utilizando-se a técnica bootstrapping, comparando a amostra original com as amostras geradas por essa técnica. Nesse sentido, foram geradas outras 500 amostras e realizado o teste $t$ Student conforme é apresentadona Tabela 3. A análise da significância dos caminhos, de acordo com Garver e Mentzer (1999), pode ser verificada a partir dos $t$-values e das cargas fatoriais das variáveis observáveis. Estes va- lores são interpretados a seguir observando a Tabela 3.

Os resultados obtidos na análise de significância dos caminhos apresentaram que a maioria destes não indicou diferença entre a amostra original e as subamostras geradas pela técnica estatística com os limites críticos para o teste $t$ de Student, no qual este teste permite a análise dos coeficientes de correlação/regressão sejam iguais a zero (HAIR et al., 20 I0). Considerou-se $t$ de Student para valores considerados significativos de $p<0,05$ e marginalmente significativos valores de $p$ entre 0,05 e 0,10 , sendo que os limites críticos para o teste $T$ com- 
TABELA 3 - Análise da significância dos caminhos

\begin{tabular}{c|l|c|c|c|c|c|c}
\hline Hipóteses & \multicolumn{1}{|c|}{ Caminho } & $\begin{array}{c}\text { Original } \\
\text { Sample (O) }\end{array}$ & $\begin{array}{c}\text { Sample } \\
\text { Mean (M) }\end{array}$ & $\begin{array}{c}\text { Erro } \\
\text { Padrão }\end{array}$ & Teste T & p value & Sig. \\
\hline H1 & $\begin{array}{l}\text { Confiança -> Propensão } \\
\text { PgMóvel }\end{array}$ & 0,558 & 0,556 & 0,048 & 11,563 & 0,000 & $* * *$ \\
\hline H2a & $\begin{array}{l}\text { Inovação Pessoal -> } \\
\text { Facilidade de uso per- } \\
\text { cebido }\end{array}$ & 0,133 & 0,133 & 0,054 & 2,439 & 0,015 & $* *$ \\
\hline H2b & $\begin{array}{l}\text { Inovação pessoal -> } \\
\text { Propensão PgMóvel }\end{array}$ & 0,134 & 0,133 & 0,056 & 2,418 & 0,016 & $* *$ \\
\hline H2c & $\begin{array}{l}\text { Inovação Pessoal -> } \\
\text { Utilidade percebida }\end{array}$ & 0,513 & 0,515 & 0,041 & 12,493 & 0,000 & $* * *$ \\
\hline H3a & $\begin{array}{l}\text { Facilidade de uso per- } \\
\text { cebido -> Propensão ao } \\
\text { PgMóvel }\end{array}$ & 0,098 & 0,098 & 0,046 & 2,127 & 0,034 & $* *$ \\
\hline H3b & $\begin{array}{l}\text { Utilidade percebida -> } \\
\text { Propensão ao PgMóvel }\end{array}$ & 0,103 & 0,105 & 0,049 & 2,097 & 0,037 & $* *$ \\
\hline H4a & $\begin{array}{l}\text { Autoeficácia -> Facilida- } \\
\text { de de uso percebido }\end{array}$ & 0,289 & 0,299 & 0,061 & 4,740 & 0,000 & $* * *$ \\
\hline H4b & $\begin{array}{l}\text { Autoeficácia -> Propen- } \\
\text { são ao PgMóvel }\end{array}$ & 0,085 & 0,089 & 0,049 & 1,747 & 0,081 & $*$ \\
\hline
\end{tabular}

Notas: ${ }^{*} p<0,10,{ }^{* *} p<0,05 \quad{ }^{* *} 0<0,01$.

Fonte: elaboração própria.

preenderam, $\mathrm{p}<0,10=1,64 ; \mathrm{p}<0,05=1,96$; e $\mathrm{P}<0,0 \mathrm{I}=2,57$ (teste bilateral $=$ nível de significância $=\mathrm{P}$ - Erro do Tipo I).A seguir são apresentadas as análises de cada um dos caminhos que compreenderam as respectivas hipóteses de pesquisa.

HI: A relação causal do caminho "Confiança" e "Propensão ao pagamento móvel" com carga original $=0,558$ foi significativa e apresentou o segundo maior teste $\mathrm{t}=||, 563$. Isto indica que o usuário demonstra preocupação com a segurança ao utilizar dispositivos móveis para serviços de pagamento, buscando informações a respeito do fornecedor e da empresa, se há alguma irregularidade legal e se está de acordo com políticas de privacidade e segurança. Neste contexto, os usuários preocupam-se em identificar dentro do histórico destas empresas reconhecimentos externos, tais como selos de certificação e regulamentações que sustentem este tipo de transações. Os usuários também buscam a confiança com base em atitudes e percepções éticas dos prestadores de serviço que sigam e cumpram regulamentações específicas.

H2a: O caminho "Inovação Pessoal” e "Facilidade de uso percebida" com carga original $=0,133$ e teste $t=2,439$ indicou significância no processo de aprendizado dos usuários em incorporar novos conhecimentos a partir de um dispositivo móvel. Portanto, isto traz uma mudança de paradigma no processo de aceitação de uma nova prática que não se traduz em modismo, mas caracteriza-se como uma tendência de mercado. $A$ inovação pessoal é uma motivação intrínsica do indivíduo em aprender, que é motivada pela facilidade do uso percebida que é a aceitação de uma nova ótica pelo processo de aprendizado.Assim, a motivação ao uso de novas tecnologias é motivada por novos conhecimentos.

H2b: A relação causal do caminho "Inovação Pessoal" e "Propensão ao pagamento móvel" com carga original=0, I 34 e teste 
$t=2,4$ I 8 apresentou significância indicando disposição pessoal dos usuários para assimilar uma tecnologia "nova". Diante disto, podemos verificar que a motivação intrínseca (pessoal) é obtida do interior do indivíduo e é estimulada pela motivação extrínseca (ambiente em constante mudança e adaptação) no que tange as novas tecnologias. Muitos usuários de serviços de pagamento móveis adotam tal prática em função das opiniões de conhecidos e/ou fontes que possam transmitir segurança.

H2c: Os construtos "Inovação Pessoal" e "Utilidade percebida" com carga original $=0,513$ e teste $t=12,493$ apresentaram a maior significância nesta pesquisa. Isso pode ser explicado que apesar das potenciais dificuldades que os usuários de pagamentos móveis possam ter em relação ao uso de novas tecnologias específicas, estes sentem-se estimulados a partir das necessidades e benefícios que possam adquirir a partir do uso destas tecnologias. Dentre os principais benefícios observados, destacam-se a interatividade; agilidade; economia de tempo; praticidade; e acesso a informações em tempo real. A motivação para o aprendizado de uma nova tecnologia de pagamento móvel surge com os potenciais benefícios que o usuário pode alcançar. Isto também poderá aumentar o rendimento das atividades e melhorar a eficácia nas atividades profissionais e pessoais.

H3a: A relação entre "Facilidade de uso percebida" e "Propensão ao pagamento móvel" com carga original $=0,098$ e teste $\mathrm{t}=2,127$ demonstrou significância pois a propensão do consumidor ao uso de dispositivos móveis para serviços de pagamento vem da necessidade de aprendizado e de adquirir novos conhecimentos. $O$ uso de uma tecnologia desconhecida é uma forma de aprender novos meios de lidar com atividades do dia-a-dia, simplificando e minimizando o tempo necessário para realização dessas atividades.

H3b: Os construtos "Utilidade percebida" e "Propensão ao pagamento móvel" com carga original $=0,103$ e teste $t=2,097$ apresentaram significância, pois os usuários distinguem os benefícios que o uso desta nova tecnologia de pagamento pode lhes trazer, suprindo suas necessidades de realização de pagamentos e minimizando o tempo, distância e trazendo praticidade a rotina. Isso pode ser explicado pelos respondentes, a maior parte dos usuários é representada por mulheres que trabalham, estudam e muitas vezes ainda precisam cuidar da casa. Tantas atividades muitas vezes diminuem o tempo para atividades necessárias (como pagamentos), portanto, o uso de dispositivos móveis em qualquer lugar para realizar essas atividades facilitam a vida do consumidor.

H4a: Ao analisar os construtos "Autoeficácia" e " Facilidade de uso percebida" com carga original $=0,289$ e teste $t=4,740$, observou-se que indicaram significância já que as habilidades necessárias para realização de uma atividade estão diretamente ligadas ao aprendizado. Ainda que para o uso destas tecnologias não sejam necessárias habilidades técnicas, elas podem ser adquiridas pelo uso da prática levando ao aprendizado. A utilização constante destas tecnologias favorece ao aprimoramento de habilidades e assim, ao conhecimento, criando um ciclo constante de necessidades, uso e conhecimento adquirido por prática.

H4b: Finalmente, a última relação entre "Autoeficácia" e "Propensão ao pagamento móvel" com carga original $=0,085$ e teste 
$\mathrm{t}=I, 747$ obteve resultado significativo no nível 0,10 . Isto caracterizou de forma geral, que os usuários não acreditam que seja necessária habilidade técnica para utilizar o serviço de pagamento em dispositivos móveis. Esse resultado pode ser explicado pois dos 365 entrevistados, 207 foram jovens abaixo de 26 anos, uma geração habituada com a tecnologia que não se preocupa em obter conhecimento técnico ou ler manuais para aprender a utilizar, aprendem na prática com a "tentativa e erro". Essa geração já nasceu em um ambiente em constante mudança em uma "nowconomy", isto, é a busca pelo imediatismo, reduzindo assim, o tempo de utilização das novas tecnologias.

\section{CONCLUSÕES}

Este estudo é uma adaptação para o Brasil do modelo de Duane, O' Reilly, e Andreev (20I4) sobre a Modelagem da propensão do consumidor ao uso de serviços de pagamentos móveis utilizando dispositivos móveis. Nesse sentido, é possível comprovar que quase todos os construtos influenciam na propensão ao uso desta tecnologia, o que pode auxiliar empresas que fornecem o serviço de pagamentos móveis a identificar estratégias para aprimorar seus serviços e atrair cada vez mais o consumidor.

O serviço de pagamento móvel apesar de já consolidado em alguns países do mundo, ainda está em crescimento no Brasil, o que pode ser explicado pela imaturidade do mercado e a falta de padrões técnicos e regulamentações, além das incertezas geradas pelas questões tecnológicas e de negócios que precisam ser melhoradas pelas empresas de telefonia móvel e instituições financeiras (ONDRUS; LYYTINEN; PIGNEUR, 2009).

Os resultados obtidos demonstraram que todas as hipóteses foram validadas no mode- lo, sendo que $7 \mathrm{com}$ valores significativos em $\mathrm{p}<0,05$ e I com valor marginalmente significativo entre 0,05 e 0,10 , no caso, o construto autoeficácia que apesar de influenciar a propensão ao uso de pagamento em dispositivos móveis, demonstrou maior atenção, dado que a maior parte dos usuários por pertencerem a grupos de jovens usuários, não considerou necessário possuir habilidades técnicas para utilizar estas tecnologias.

A privacidade, o histórico das operações, a regulamentação e a ética do fornecedor do serviço são importantes e influenciam na escolha ou não pelo uso do serviço, que precisa se sentir seguro ao escolher utilizar o serviço de pagamento móvel. A "Inovação Pessoal" e a "Facilidade de uso percebida" são diretamente relacionadas já que a primeira vem da disposição interior do indivíduo e é motivada pela segunda que é uma nova ótica, já que pelo conhecimento o usuário passa a ter outra percepção em relação ao serviço de pagamento por meio de dispositivos móveis.

O construto "Confiança" teve a segunda maior significância em relação a propensão ao pagamento móvel, demonstrando como o usuário se preocupa com a segurança em relação aos seus dados, já que para estas operações é necessário fornecer dados pessoais e de pagamento.

No caso da "Inovação Pessoal" e da "Propensão ao uso de pagamentos móveis" são duas motivações que influenciam na decisão do consumidor. A motivação interior em aprender e buscar novos conhecimentos, e a motivação exterior que seria a curiosidade em conhecer uma nova tecnologia. Assim, o ambiente em constante mudança e o aparecimento de novas tecnologias motiva o indivíduo a buscar esse conhecimento.

Outro motivador externo seria a "Uti- 
lidade percebida" em relação a "Inovação pessoal”, já que ao perceber os benefícios que podem ser gerados pelo uso desta tecnologia, como a redução do tempo para atividades, acesso e transferência de informação, agilidade e a praticidade, o consumidor se sente motivado a aprender a utilizar para poder também se beneficiar com seu uso.

A "Facilidade de uso percebida" se relaciona com a "Propensão ao pagamento móvel" pois o consumidor ao começar a utilizar esta tecnologia adquire novos conhecimentos em relação ao dispositivo já que aprende com o uso constante e aprimora seu modo de usar e lidar com esse serviço.

Os benefícios gerados pelo uso do serviço de pagamento móvel são o que chamam a atenção do consumidor a tentar utilizar essa tecnologia. Por isso a relação entre "Utilidade Percebida" e "Propensão ao pagamento móvel" teve significância, já que o usuário busca um produto ou serviço que atenda suas necessidades, caso encontre ele passa a utilizar e se beneficiar como no caso do serviço de pagamento em dispositivos móveis, já que com essa praticidade ele pode realizar suas atividades aonde quer que esteja.

A "Autoeficácia" e a "Facilidade de uso percebida" têm uma relação direta, pois a primeira é a habilidade em executar algum comportamento (DABHOLKAR; BAGOZZl, 2002), e a segunda é o aprendizado gerado por novos conhecimentos. $O$ consumidor que passa a utilizar o serviço de pagamento móvel obtém conhecimento que gera a habilidade necessária para o uso desta tecnologia.

\section{Recomendações para as empresas que fornecem serviços de pagamento móvel}

Dentre os construtos avaliados e a realidade do negócio, foi observado que para que esta tecnologia possa crescer no mercado, as organizações precisam buscar cada vez mais a confiança do consumidor. No caso do serviço de pagamento móvel, as empresas que fornecem esse serviço ou tem alguma interação essencial para a realização deste, são as empresas de cartão de crédito, operadoras de telefonia móvel e instituições financeiras como bancos.

Ainda que sejam empresas já consolidadas no mercado, esta nova tecnologia ainda não o é, e muitas vezes o usuário não sabe quem está por trás do serviço ou produto e a credibilidade da organização não é levada em conta no caso já que a informação não é divulgada.

Os serviços com novas tecnologias de pagamento móvel prometem substituir em alguns anos os tão populares cartões de plástico. Para o consumidor, os serviços poderão ser remotos por troca de mensagens ou pagamento presencial sem contato com outras máquinas. Nesse sentido, o trabalho empreendido pelas empresas que compõe essa cadeia, no entanto, revela uma realidade complexa, pois atualmente não há um padrão que predomine no mercado global.

Um modo de ganhar a confiança do usuário e atrair novos clientes é divulgar o máximo de informações possível em relação ao produto, na página deste, no aplicativo, em sua propaganda ou qualquer outro meio viável. $A$ informação faz com que o consumidor se sinta mais confiante e confortável para utilizar uma tecnologia sabendo que seus dados não serão utilizados de forma errada.

Investimentos em propaganda e mensagens ao usuário, por exemplo, 
dentro do aplicativo do dispositivo móvel, pode instigar a curiosidade. Já que se esta em um ambiente tecnológico, as organizações precisam aproveitar os recursos enquanto o usuário utiliza $\circ$ serviço para mostrar que sim, é necessária essa habilidade.
O que as organizações precisam atentar é que não basta apenas entrar no mercado da tecnologia, mas é necessário acompanhar as tendências e o perfil dos consumidores, em função de ser um mercado dinâmico, assim o modo de gerir esse negócio irá promover o seu uso. 


\section{REFERÊNCIAS}

AGARWAL, R.; PRASAD, J.A conceptual and operational definition of personal innovativeness in the domain of information technology. Information Systems Research, 9 (2), I998, p. 204-2I 5.

BAUER, H. H.; BARNES, S. J., REICHARDT, T.; NEUMANN, M. M. Driving consumer acceptance of mobile marketing: a theoretical framework and empirical study. Journal of Electronic Commerce Research, 6 (3), 2005 , p. $181-192$.

BROWN, S. A.; VENKATESH, V. Model of Adoption of Technology in the Household: A Baseline Model Test and Extension Incorporating Household Life Cycle. MIS Quarterly. (29:4), 2005, p. 399426.

BROWNE, M.W.; CUDECK, R. Alternative ways of assessing model fit. In: K. A. Bollen; J. S. Long (Eds.), Testing structural equation models. Beverly Hills, CA: Sage, 1993, p. I36-162.

CHAU, P.Y. K.; HU, P. J. H.; LEE, B. L. P., AU, A. K. K. Examining customers' trust in online vendors and their dropout decisions: an empirical study. Electronic Commerce Research and Applications, 6 (2), 2007, p. I7|-182.

CHEN, K., CHEN, J.V.; YEN, D. C. Dimensions of self-efficacy in the study of smart phone acceptance. Computer Standards and Interfaces, 33 (4), 20 I I, p. 422-43I.

CHIN,W.W.The Partial Least Squares approach to structural equation modeling. In: MAR-COULIDES, G.A. (Ed.). Modern Methods for business research. USA: Lawrence Erlbaum Associates, 1994, p. 295-336.

CHIU, C. M.; LIN, H. Y.; SUN, S. Y.; HSU, M. H. Understanding custo- mers' loyalty intentions towards online shopping: an integration of technology acceptance model and fairness theory. Behavior and Information Technology, 28 (4), 2009, p. 347-360.

CLEFF, E. B. Implementing the legal criteria of meaningful consent in the concept of mobile advertising. Computer Law and Security Report, 23 (3), 2007, p. 262-269. DABHOLKAR, P. A.; BAGOZZI, R. P. An attitudinal model of technology-based selfservice: Moderating effects of consumer traits and situational factors. Journal of the Academy of Marketing Science, 30, 2002, p. 318-34I.

DAHLBERG, T.; MALLAT, N.; ONDRUS, J.; ZMIJEWSKA,A. Mobile payment market and research - past, present and future, in: Presentation at Helsinki Mobility Roundtable, Helsinki, Finland, June I-2, 2006.

DAHLBERG, T.; MALLAT, N.; ONDRUS, J.; ZMIJEWSKA, A. Past, present and future of mobile payments research: a literature review. Electronic Commerce Research and Applications, 7 (2), 2008, p. I65-I8I.

DEWAN, S. G.; CHEN, L. Mobile payment adoption in the USA: a cross industry, cross-platform solution. Journal of Information Privacy and Security, I (2), 2005, p. 4-28.

DUANE, A.; O'REILLY, P.; ANDREEV, P. Realising M-Payments: modelling consumers' willingness to M-pay using Smart Phones. Behaviour \& Information Technology, 20 I4, p. 3 I8-334. DOI: I0.1080/0 | 44929X.20I2.745608.

FAUL, F. Statistical power analyses using G*Power 3.I: Tests of correlation and regression analyses.
Behavior Research Methods, 4I (4), 2009, p. II 49-I I60.

FORNEL, C.; LARCKER, D. F. Evaluating structural equation models with unobservable, variables and measurement error. Journal of Marketing Research, v.l8, n.I, I981, p. 39-50.

GARVER, M. S.; MENTZER, J.T. Logistics research methods: Employing structural equation modeling to test for construct validity, Journal of Business Logistics, 20, I, 1999, p. 33-57.

GUPTA, S., XU, H.; ZHANG, X. Balancing privacy concerns in the adoption of location-based services: an empirical analysis. International Journal of Electronic Business, 9 (I/2), 20 I I, p. I I8-I37.

HAIR, J. F.; BLACK,W.C.; BABIN, B. J.; ANDERSON, R. E. Multivariate Data Analisys. $7^{\text {th }}$ Edition. NJ: Prentice Hall, 2010.

HSU, H. Y. S.; KULVIWAT, S. An integrative framework of technology acceptance model and personalization in mobile commerce. International Journal of Technology Marketing, I (4), 2006, p. 393-410.

HSU, M. H.; CHANG, C. M.; YEN, C.H. Exploring the antecedents of trust in virtual communities. Behaviour and Information Technology, 30 (5), 20II, P. 587-60I.

HSU, M. H.; JU, T. L.; YEN, C. H.; CHANG, C. Knowledge sharing behavior in virtual communities: the relationship between trust, self-efficacy, and outcome expectations. International Journal of Human-Computer Studies, 65, 2007, p. I53-169.

HU, WEN-CHEN; LEE, CHUNG -WEl; KOU,WEIDONG. Advances in security and payment 
methods for Mobile Commerce. Londres: Idea Group Publishing, 2005.

IM, I., KIM, Y.; HAN, H. J. The effects of perceived risk and technology type on users' acceptance of technologies. Information \& Management, 45 (I), 2008, p. I-9.

BRYNJOLFSSON, E.; HU Y. J.; RAHMAN, M. S. Competing in the Age of Omnichannel Retailing, MIT Sloan Management Review (54:4), 20I3, 20I3, p. 23 29.

LEE, C. C.; HSIEH, M. C.; HUANG, H. C. The influence of mobile self-efficacy on attitude towards mobile advertising. AISS: Advances in Information Sciences and Service Sciences, 3 (3), 20 I I, p. 100-108.

MALHOTRA, NARESH K. Essencials of Marketing Research: A Hands-on Orientation. Prentice Hall, I Edition, January 20, 2014

ONDRUS, J.; LYYTINEN, K.; PIGNEUR, Y. Why mobile payments fail? Towards a dynamic and multi-perspective explanation. Proceedings of the 42nd Hawaii International Conference on System Sciences, Waikoloa, Hawaii, 2009.

ONDRUS, J.; LYYTINEN, K. Mobile payments market: towards another clash of the titans. Proceedings of the Tenth International Conference of Mobile Business (ICMB), 20I I.

PARASURAMAN, V.; ZEITHAMI, V.A.; BERRY, L. L. A conceptual model of service quality and its implications for future research. Journal of Marketing, v.49, n.4, 1985, p.
4I-50, fall.

PEREZ, Gilberto; ROSA, Aline Dalla; MEDEIROS, Alberto Jr:; COMAR, Michelle. (20/3). Trends in the Use of Mobile Payment in Brazil: An Analysis Using the Delphi Method. CONF-IRM 20I3 Proceedings. Paper 49.

ROCA, J. C.; GARCIA, J.J.; de laVEGA, J. J. The importance of perceived trust, security and privacy in online trading systems. Information Management \& Computer Security, I7 (2), 2008, p. 96-II3. SACCOL, Amarolinda Zanela.; REINHARD, Nicolau. Tecnologias de Informação Móveis, Sem fio e Ubíquas: Definições, Estado-da-Arte e Oportunidades de Pesquisa. Revista de Administração Contemporânea, v. II, n. 4, Out./ Dez., 2007, p. I75- 198.

SANCHEZ-FRANCO, M.; RONDAN-CATALUÑA, F. J. Connection between customer emotions and relationship quality in online music services. Behaviour \& Information Technology, 29 (6), 20I I, p. 633-65I.

SCHIERZ, P. G.; SCHILKE, O.;WIRTZ, B.W. Understanding consumer acceptance of mobile payment services: an empirical analysis. Electronic Commerce Research and Applications, 9 (3), 20 I 0, p. 209-2I6.

SLADE, Emma; WILLIAMS, Michael; DWIVDEl, Yogesh. Extending UTAUT2 to Explore Consumer Adoption of Mobile Payments. UK Academy for Information Systems Conference Proceedings 2013 , Paper 36, 2013.

STREINER, D. L. Being inconsistent about consistency: when coeffi- cient alpha does and doesn't matter. Journal of Personality Assesment. v.80, 2003, p. 21 7-222.

THEOHARIDOU, Marianthi.; MYLONAS,Alexios; GRITZALIS, Dimitris. A Risk of Assessment Method for Smartphones. Athens: Athens University of Economics and Business (AUEB), 2013.

UZUREAU, C.; NEWTON, A. Hype Cycle for Financial Services Payment Systems. Gartner, 20I I. ID: G002I 4036

VARNALI, K.; TOKER, A. Mobile marketing research: the state of the art. International Journal of Information Management, 30, 2009, p. |44-I5I.

VIEHLAND, D.; YOONG LEONG, R. $\mathrm{S}$. Consumer willingness to use and pay for mobile payment services. International Journal of Principles and Applications of Information Science and Technology, 3 (I), 20I0, p. 34-46.

WU, J. J.; TSANG, A. S. L. Factors affecting members' trust belief and behaviour intention in virtual communities. Behaviour and Information Technology, 27 (2), 2008, p. II 5-I 25.

YANG, M. H.; LIN, B.; CHANDLREES, N.; CHAO, H. Y. The effect of perceived ethical performance of shopping websites on consumer trust. Journal of Computer Information Systems, 50 (I), 2009, p. 15-24.

YOUNG HOON, K.; KIM, D. J.; YUJONG, H. Exploring online transaction self-efficacy in trust building in B2C E-commerce. Journal of Organizational and End User Computing, 2I (I), 2009, p. 37-59. 